\title{
A New Sine-Gordon Equation Expansion Algorithm to Investigate some Special Nonlinear Differential Equations
}

\author{
Zhenya Yan \\ Mathematics Mechanization Key Laboratory \\ Academy of Mathematics and Systems Science \\ Chinese Academy of Sciences \\ Beijing 100080, China \\ zyyan@mmrc.iss.ac.cn
}

\begin{abstract}
.
A new transformation method is developed using the general sine-Gordon travelling wave reduction equation and a generalized transformation. With the aid of symbolic computation, this method can be used to seek more types of solutions of nonlinear differential equations, which include not only the known solutions derived by some known methods but new solutions. Here we choose the double sine-Gordon equation, the Magma equation and the generalized Pochhammer-Chree(PC) equation to illustrate the method. As a result, many types of new doubly periodic solutions are obtained. Moreover when using the method to these special nonlinear differential equations, some transformations are firstly needed. The method can be also extended to other nonlinear differential equations.
\end{abstract}

Keywords: Sine-Gordon equation; nonlinear differential equation; symbolic computation; doubly periodic solution; solitary wave solution

\section{Introduction}

It may be difficult to seek directly solutions of nonlinear differential equations in nonlinear science because of the complexity of theirs. It is a natural way to establish relationships among some simple and solvable equations and many complexly nonlinear equations. These relationships and the known solutions of these simple and solvable equations can be used to get the corresponding solutions of other complexly nonlinear differential equations.

From the famous sine-Gordon equation

$$
\frac{\partial^{2} u}{\partial x \partial t}=\alpha \sin u
$$

which appears in many branches of nonlinear science[1], one can use the travelling wave transformation $u(\xi)=2 w(\xi), \xi=k(x-\lambda t), b=\alpha /(k \lambda)$ to reduces (1.1) to the first-order ODE

$$
d w(\xi) /(d \xi)=\mu \sqrt{a+b \sin ^{2} w(\xi)}, \quad \mu= \pm 1,
$$

where $a$ is an integration constant. For different parameters $a, b,(1.2)$ may possess abundant different solutions. To use solutions of (1.2) to express the corresponding solutions of other 
nonlinear differential equations, the first step is to seek some solutions of (1.2), which may be simple to solve. We have the following proposition:

Proposition. The general sine-Gordon travelling wave reduction equation (1.2) possesses these solutions in the following cases:

(1) In the case $a=0, b=1,(1.2)$ reduces to the first-order ODE

$$
d w(\xi) /(d \xi)=\sin w(\xi),
$$

which has the solutions:

$$
\sin [w(\xi)]=\operatorname{sech}(\xi), \quad \text { or } \quad \cos [w(\xi)]=-\tanh (\xi) .
$$

and

$$
\sin [w(\xi)]=i \operatorname{csch}(\xi), \quad \text { or } \quad \cos [w(\xi)]=-\operatorname{coth}(\xi),
$$

where $i=\sqrt{-1}$.

(2) In the case $a=1, b=-m^{2},(1.2)$ becomes the first-order ODE

$$
d w(\xi) /(d \xi)= \pm \sqrt{1-m^{2} \sin ^{2} w(\xi)}
$$

where $m$ is the modulus of Jacobi elliptic functions[2,3], which has the solutions

$$
\sin [w(\xi)]=\operatorname{sn}(\xi ; m), \quad \text { or } \quad \cos [w(\xi)]=\operatorname{cn}(\xi ; m),
$$

and

$$
\sin [w(\xi)]=\mathrm{ns}(\xi ; m) / m, \quad \text { or } \quad \cos [w(\xi)]=i \mathrm{ds}(\xi ; m) / m,
$$

(3) In the case $a=m^{2}, b=-1,(1.2)$ becomes the first-order ODE

$$
d w(\xi) /(d \xi)= \pm \sqrt{m^{2}-\sin ^{2} w(\xi)},
$$

which has the solutions

$$
\sin [w(\xi)]=m \operatorname{sn}(\xi ; m), \quad \text { or } \quad \cos [w(\xi)]=\operatorname{dn}(\xi ; m) .
$$

and

$$
\sin [w(\xi)]=\mathrm{ns}(\xi ; m), \quad \text { or } \quad \cos [w(\xi)]=i \operatorname{cs}(\xi ; m)
$$

Remark 1. We may obtain other types of solutions of (1.2), which will lead to other kinds of nonlinear differential equations.

According to the solution (1.4) of (1.3), the transformation

$$
u(\xi)=u(w(\xi))=A_{0}+\sum_{j=1}^{n} \cos ^{j-1} w\left[A_{j} \sin w+B_{j} \cos w\right]
$$


was proposed to seek solitary waves of many nonlinear evolution equations[4-6]. Obviously (1.3) is a special case of (1.2). This may lead to obtain only solitary wave solutions using (1.4) and (1.12). In fact, we can also use the solution (1.5) of (1.3) and (1.12) to seek the singular solitary wave solutions of nonlinear wave equations.

Recently, Fu et al[7] used the same transformation (1.12) and the only solution (1.10) of (1.9) to seek some doubly periodic solutions of some nonlinear evolution equations in the form

$$
u(\xi)=A_{0}+\sum_{i=1}^{n} \operatorname{dn}^{i-1}(\xi ; m)\left[A_{i} m \operatorname{sn}(\xi ; m)+B_{i} \operatorname{dn}(\xi ; m)\right] .
$$

In fact the formal solution is equivalent to a special case of our extended Jacobi elliptic function method $[8,9]$.

Moreover we can also apply solutions (1.7), (1.8) of (1.6) or another solution (1.11) of (1.9) and (1.12) to search for more types of solitary wave solutions and doubly periodic solutions of nonlinear evolution equations.

To use solutions of (1.2) to construct more types of nonlinear differential equations, we would like to improve the transformation (1.12) to be new form, and then use the new form and three special cases (1.3), (1.6) and (1.9) of (1.2) to seek more types of new solitary wave solutions and doubly periodic solutions of nonlinear wave equations.

\section{The new sine-Gordon equation expansion method}

Consider the following nonlinear evolution equation, say, in two variables $x, t$

$$
F\left(u, u_{t}, u_{x}, u_{x x}, u_{x t}, u_{t t}, \ldots\right)=0,
$$

we seek its travelling wave solution of physical significance, if available, in the form $u(x, t)=$ $u(\xi), \xi=k(x+\lambda t)$. We assume that $(2.1)$ has the solution in the form

$$
u(\xi)=A_{0}+\sum_{i=1}^{n} \frac{\cos ^{i-1} w(\xi)}{[R+P \sin w(\xi)+Q \cos w(\xi)]^{i}}\left[A_{i} \sin w(\xi)+B_{i} \cos w(\xi)\right],
$$

where $A_{i}(i=0,1, \ldots, n), B_{j}(j=1,2, \ldots, n), R^{2}+P^{2}+Q^{2} \neq 0$ are constants to be determined later and $w=w(\xi)$ satisfies $(1.2)$

According to (1.2) and (2.2), we define a polynomial degree function as $D(u(w))=n$, thus we have

$$
D\left(u^{p}(w)\left(d^{s} u(w) /\left(d \xi^{s}\right)\right)^{q}\right)=n p+q(n+s) .
$$

Therefore we can determine the parameter $n$ by balancing the highest order derivative term with nonlinear terms in (2.1). The method is summed up as follows:

Step i: Reduce the given nonlinear partial differential equation (2.1) to an ordinary differential equation (ODE) using the travelling wave transformation $u(x, t)=u(\xi), \xi=$ $k(x+\lambda t)$.

Step ii: Fix the parameter $n$ in (2.2) by balancing the highest order derivative terms and nonlinear terms and thus give the formal solution (2.2) (Remark: If $n$ is not a positive integers, then we need to make the transformation $\left.u=v^{n}\right)$. 
Step iii: Substitute (2.2) with the fixed parameter $n$ along with (1.2) into the obtained ODE to yield the polynomial equation in $w^{\prime s} \sin ^{i} w \cos ^{j} w$.

Step iv: Set to zero the coefficients of $w^{\prime s} \sinh ^{i} w \cosh ^{j} w(i=0,1 ; s=0,1 ; j=0,1,2, \ldots)$ to get a set of algebraic equations $C_{i j s}\left(R, P, Q, A_{s}, B_{s}\right)=0$ with respect to the unknowns $k, \lambda, R, P, Q, A_{j}(j=0,1, \ldots, n)$ and $B_{j}(j=1,2, \ldots, n)$.

Step v: Solve the set of algebraic equations, which may not be consistent to derive solitary wave solutions and doubly periodic solutions of the given nonlinear equations using $u(x, t)=u(\xi), \xi=k(x-\lambda t)$ and the known solution (1.4),(1.5), (1.7),(1.8),(1.10) and (1.11).

Remark 2. (1) Since when $m \rightarrow 1, \operatorname{sn}(\xi ; \mathrm{m}) \rightarrow \tanh \xi$ and $\operatorname{cn}(\xi ; \mathrm{m}), \operatorname{dn}(\xi ; \mathrm{m}) \rightarrow \operatorname{sech} \xi$; while when $m \rightarrow 0, \operatorname{sn}(\xi ; \mathrm{m}) \rightarrow \sin \xi, \operatorname{cn}(\xi ; \mathrm{m}) \rightarrow \cos \xi$ and $\operatorname{dn}(\xi ; \mathrm{m}) \rightarrow 1$, thus it is easy to see that the method is used to obtain both solitary wave solutions and Jacobi elliptic function solutions.

(2) It is easy to see that the solutions derived from the above-mentioned method include both the results of the sine-cosine method[4-6], the Riccati method[10-12], the Fu's method[7], the sinh-Gordon equation expansion method[13], the algebraic method without Weierstrass elliptic function solutions[14], the projective Riccati equation method[15,16] and the sn- and cn-function method[17] and new solutions.

Obviously, the method is applied to the nonlinear differential equation (2.1) with $F$ being a polynomial in $u$ and its derivatives. Otherwise we need to make some transformations to reduce $(2.1)$ to a polynomial equation in new variables and its derivative.

If a certain nonlinear differential equation does not possess doubly periodic solutions, then we may use (2.2) and (1.3) to seek its solitary wave solutions. In the following we mainly use (2.2) and (1.6) or (1.9) to seek solitary wave solutions and doubly periodic solutions of some special nonlinear wave equations which are needed to be modified, when the method in Section 2 is used to seek their solutions.

\section{Applications}

\subsection{The double sine-Gordon equation}

$$
u_{x t}=\sin u+\sin 2 u,
$$

which appears in many physical branches[1]. Some solutions of (3.1) has been given[18]. Here we will seek new solutions of (3.1).

If we make the travelling wave transformation $u(x, t)=u(\xi), \xi=k(x+\lambda t)$, then (3.1) reduces to

$$
k \lambda \frac{d^{2} u}{d \xi^{2}}-\sin u-2 \sin 2 u=0 .
$$

which is not a polynomial in $u$. If we make the transformation $u(\xi)=2 \arctan v$, then (3.2) becomes

$$
k \lambda\left(1+v^{2}\right) \frac{d^{2} v}{d \xi^{2}}-2 k \lambda v\left(\frac{d v}{d \xi}\right)^{2}-3 v+v^{3}=0 .
$$

According to Step 2, we know that $n=1$ in (2.2) and thus suppose that (3.1) has the 
solution in the form

$$
v(\xi)=A_{0}+\frac{A_{1} \sin w(\xi)}{R+P \sin w(\xi)+Q \cos w(\xi)}+\frac{B_{1} \cos w(\xi)}{R+P \sin w(\xi)+Q \cos w(\xi)},
$$

and $w(\xi)$ satisfying (1.2).

With the aid of Maple, substituting (3.3) into (3.2) along with (1.2), we have the polynomial of $\sin ^{i} w \cos ^{j} w(i=0,1 ; j=0,1,2, \ldots)$. Setting their coefficients to zero yields a set of algebraic equations (We omit them here).

By solving the set of algebraic equations, we can fix those parameters. Therefore we have the following doubly periodic solutions of (3.1) from (3.4) and the solutions (1.7),(1.8), (1.10) or (1.11) of (1.2):

From (3.4) and (1.6), we have the doubly periodic solutions of (3.1)

$$
u_{1}=2 \arctan \left\{\frac{\operatorname{sn}[k(x+\lambda t) ; m]}{ \pm R+R \operatorname{cn}[k(x+\lambda t) ; m]}\right\}
$$

where

$$
\begin{gathered}
R=\sqrt{1 / 3\left(1-2 m^{2} \pm 2 \sqrt{m^{4}-m^{2}+1}\right)}, \quad \lambda=-\left(1+3 R^{2}\right)\left[k m^{2}\left(1+R^{2}\right)\right] . \\
u_{2}=2 \arctan \left\{A_{1} \operatorname{sn}[k(x+\lambda t) ; m]\right\},
\end{gathered}
$$

where

$$
\begin{gathered}
A_{1}=\sqrt{6 m^{2} /\left[-\left(1+m^{2}\right) \pm \sqrt{m^{4}+14 m^{2}+1}\right]}, \quad \lambda=-1 /\left[k\left(1+m^{2}+2 m^{2} / A_{1}^{2}\right)\right] . \\
u_{3}=2 \arctan \left\{A_{1} \operatorname{sc}[k(x+\lambda t) ; m]\right\},
\end{gathered}
$$

where

$$
\begin{gathered}
A_{1}=\sqrt{1 / 2\left(m^{2}-2 \pm \sqrt{m^{4}-16 m^{2}+16}\right)}, \quad \lambda=\left(A_{1}^{2}+3\right) /\left[k m^{2}\left(A_{1}^{2}-1\right)\right] . \\
u_{4}=2 \arctan \left\{\frac{\operatorname{cn}[k(x+\lambda t) ; m]}{ \pm R+R \operatorname{sn}[k(x+\lambda t) ; m]}\right\},
\end{gathered}
$$

where

$$
\begin{gathered}
R=\sqrt{\left[-\left(1+m^{2}\right) \pm 2 \sqrt{m^{4}-m^{2}+1}\right] /\left(3\left(m^{2}-1\right)\right)}, \quad \lambda=\left(3 R^{2}+1\right) /\left(k m^{2}\left(1+R^{2}\right)\right) . \\
u_{5}=2 \arctan \left\{B_{1} \operatorname{cn}[k(x+\lambda t) ; m]\right\},
\end{gathered}
$$

where

$$
\begin{gathered}
B_{1}=\sqrt{6 m^{2} /\left[1-2 m^{2} \pm \sqrt{16 m^{4}-16 m^{2}+1}\right]}, \quad \lambda=1 /\left[k\left(2 m^{2}-1+2 m^{2} / B_{1}^{2}\right] .\right. \\
u_{6}=2 \arctan \{1 / P \operatorname{cs}[k(x+\lambda t) ; m]\},
\end{gathered}
$$


where

$$
P=\sqrt{1 / 6\left[\left(2-m^{2}\right) \pm \sqrt{m^{4}-16 m^{2}+16}\right]}, \quad \lambda=-\left(3 P^{2}+1\right) /\left[k m^{2}\left(P^{2}-1\right)\right] .
$$

Remark 3. When $m \rightarrow 1$ in (3.5), we have the solitary wave solution

$$
u_{1}^{\prime}=2 \arctan \left\{\frac{\sqrt{3} \tanh [k(x-3 /(2 k) t)]}{\operatorname{sech}[k(x-3 /(2 k) t)] \pm 1}\right\} .
$$

When taking the sign,$+(3.11)$ is a regular solitary wave solution, while taking the sign -, (3.11) is a singular solitary wave solution.

In addition, if we use (3.4) and (1.9) to investigate (3.3), then we have

$$
u_{7}=2 \arctan \left\{\frac{m \operatorname{sn}[k(x+\lambda t) ; m]}{ \pm R+R \operatorname{dn}[k(x+\lambda t) ; m]}\right\}
$$

where

$$
\begin{gathered}
R=\sqrt{1 /\left(3 m^{2}\right)\left[m^{2}-2 \pm 2 \sqrt{m^{4}-m^{2}+1}\right]}, \quad \lambda=-\left(1+3 R^{2}\right) /\left[k\left(R^{2}+1\right)\right] . \\
u_{8}=2 \arctan \{m \operatorname{sd}[k(x+\lambda t) ; m]\},
\end{gathered}
$$

where

$$
\begin{gathered}
A_{1}=\sqrt{1 /\left(2 m^{2}\right)\left[1-2 m^{2} \pm \sqrt{16 m^{4}-16 m^{2}+1}\right]}, \quad \lambda=-\left(A_{1}^{2}+3\right) /\left[k\left(A_{1}^{2}-1\right)\right] . \\
u_{9}=2 \arctan \left\{\frac{\operatorname{dn}[k(x+\lambda t) ; m]}{ \pm R+R m \operatorname{sn}[k(x+\lambda t) ; m]}\right\},
\end{gathered}
$$

where

$$
\begin{gathered}
R=\sqrt{3\left(m^{2}-1\right) /\left[m^{2}+1 \pm \sqrt{m^{4}-m^{2}+1}\right]}, \quad \lambda=\left(3 R^{2}+1\right) /\left[k\left(R^{2}-1\right)\right] . \\
u_{10}=2 \arctan \{1 /(m P) \operatorname{ds}[k(x+\lambda t) ; m]\},
\end{gathered}
$$

where

$$
P=\sqrt{1 /\left(6 m^{2}\right) /\left[2 m^{2}-1 \pm \sqrt{16 m^{4}-16 m^{2}+1}\right]}, \quad \lambda=\left(3 R^{2}+1\right) /\left[k\left(R^{2}-1\right)\right] .
$$

Remark 4. According to other solutions of (1.2), we may obtain other types of solutions of (3.1). Moreover when $m \rightarrow 0$, we can obtain the corresponding periodic solutions of (3.1) from (3.4) and solution (1.7) of (1.6). We omit them here. 


\subsection{The Magma equation}

$$
u_{t}=\left[u^{n}\left(u^{-m} u_{t}\right)_{x}-u^{n}\right]_{x},
$$

where $u(x, t)$ is mean volume fraction of liquid phase which should be non-negative for any $x$ and $t$. The exponents $m$ and $n$ denote the dependency of permeability and effective viscosity characterizing thee rate of matrix compaction and distension on $u[19]$.

For differential parameters $m$ and $n$, some exact solutions of (3.16) has been obtained[19,20]. In the following we consider the case $m=0, n=4$, in which (3.16) reduces to

$$
u_{t}=u^{4} u_{x x t}+4 u^{3} u_{x} u_{x t}-4 u^{3} u_{x}
$$

We seek the travelling wave solution $u(x, t)=u(z), z=k(x+\lambda t)$. Then (3.17) becomes

$$
k^{2} \lambda\left(\frac{d u}{d z}\right)^{2}-u+\lambda u^{-2}+a u^{-3}+b=0,
$$

where $a, b$ are integration constants.

If we introduce the new independent variable $\xi$ defined by

$$
\xi=\int^{z} u^{-3 / 2}(z) d z
$$

then (3.18) can be rewritten as

$$
k^{2} \lambda\left(\frac{d u}{d \xi}\right)^{2}-u^{4}+b u^{3}+\lambda u+a=0
$$

According to Step 2, we assume that (3.20) has the solution

$$
u(\xi)=A_{0}+\frac{A_{1} \sin w(\xi)}{R+P \sin w(\xi)+Q \cos w(\xi)}+\frac{B_{1} \cos w(\xi)}{R+P \sin w(\xi)+Q \cos w(\xi)},
$$

with $w$ satisfying (1.2).

The substitution of (3.21) into (3.20) with (1.6) yields a set of algebraic equations in unknowns $A_{1}, B_{1}, A_{0}, R, P, Q, k, \lambda, a, b$. By solving the set of equations, we get the doubly periodic solutions of $(3.16)$

$$
u_{1}(\xi)=\frac{3 \sqrt{3 /\left(-4+8 m^{2}\right)}}{k^{2}\left(-1+2 m^{2}\right)} \frac{\operatorname{sn}(\xi ; m)}{R \pm R \operatorname{cn}(\xi ; m)}-\frac{3}{2 k^{2}\left(-1+2 m^{2}\right)},
$$

where $\lambda=27 /\left[k^{6}\left(-1+2 m^{2}\right)^{3}\right]$, and the relationship between $z$ and $\xi$ is $z=\int^{\xi} u_{1}^{3 / 2}(\xi) d \xi$.

$$
u_{2}(\xi)=\frac{3 m}{k^{2}\left(m^{2}+1\right) \sqrt{8\left(m^{2}+1\right) / 3}} \operatorname{sn}(\xi ; m)-\frac{3}{4 k^{2}\left(m^{2}+1\right)},
$$

where $\lambda=27 /\left[8 k^{6}\left(m^{2}+1\right)^{3}\right]$, and the relationship between $z$ and $\xi$ is $z=\int^{\xi} u_{2}^{3 / 2}(\xi) d \xi$.

$$
u_{3}(\xi)=\frac{1}{k^{2}\left(m^{2}-2\right)} \sqrt{\frac{27\left(m^{2}-1\right)}{16-8 m^{2}}} \operatorname{sc}(\xi ; m)-\frac{3}{4 k^{2}\left(m^{2}-2\right)},
$$


where $\lambda=27 /\left[8 k^{6}\left(m^{2}-2\right)^{3}\right]$, and the relationship between $z$ and $\xi$ is $z=\int^{\xi} u_{3}^{3 / 2}(\xi) d \xi$.

$$
u_{4}=\frac{3 \sqrt{\left(3 m^{2}-3\right) /\left(4 m^{2}+4\right)}}{k^{2}\left(m^{2}+1\right)} \frac{\operatorname{cn}(\xi ; m)}{R \pm R \operatorname{sn}(\xi ; m)}+\frac{3}{2 k^{2}\left(m^{2}+1\right)},
$$

where $\lambda=-27 /\left[k^{6}\left(1+m^{2}\right)^{3}\right]$, and the relationship between $z$ and $\xi$ is $z=\int^{\xi} u_{4}^{3 / 2}(\xi) d \xi$.

$$
u_{5}=\frac{3 m \sqrt{3 /\left(16 m^{2}-8\right)}}{k^{2}\left(2 m^{2}-1\right)} \operatorname{cn}(\xi ; m)+\frac{3}{4 k^{2}\left(-1+2 m^{2}\right)},
$$

where $\lambda=-27 /\left[8 k^{6}\left(2 m^{2}-1\right)^{3}\right]$, and the relationship between $z$ and $\xi$ is $z=\int^{\xi} u_{5}^{3 / 2}(\xi) d \xi$.

$$
u_{6}=\frac{3 \sqrt{3 /\left(8 m^{2}-4\right)}}{k^{2}\left(m^{2}-2\right)}[\operatorname{sn}(\xi ; m) \pm i \operatorname{cn}(\xi ; m)]+\frac{3}{2 k^{2}\left(m^{2}-2\right)},
$$

where $\lambda=-27 /\left[k^{6}\left(m^{2}-2\right)^{3}\right]$, and the relationship between $z$ and $\xi$ is $z=\int^{\xi} u_{6}^{3 / 2}(\xi) d \xi$.

In addition, the substitution of (3.21) into (3.20) with (1.9) yields a set of algebraic equations in unknowns $A_{1}, B_{1}, A_{0}, R, P, Q, k, \lambda, a, b$. By solving the set of equations, we get also the doubly periodic solutions of (3.16):

$$
u_{7}=\frac{3 m^{2} \sqrt{3 /\left(8-4 m^{2}\right)}}{k^{2}\left(m^{2}-2\right)} \frac{\operatorname{sn}(\xi ; m)}{R \pm R \operatorname{dn}(\xi ; m)}+\frac{3}{2 k^{2}\left(m^{2}+1\right)},
$$

where $\lambda=-27 /\left[k^{6}\left(m^{2}-2\right)^{3}\right]$, and the relationship between $z$ and $\xi$ is $z=\int^{\xi} u_{7}^{3 / 2}(\xi) d \xi$.

$$
u_{8}=\frac{3 \sqrt{3 /\left(16-8 m^{2}\right)}}{k^{2}\left(m^{2}-2\right)} \operatorname{dn}(\xi ; m)-\frac{3}{4 k^{2}\left(m^{2}-2\right)},
$$

where $\lambda=27 /\left[8 k^{6}\left(m^{2}-2\right)^{3}\right]$, and the relationship between $z$ and $\xi$ is $z=\int^{\xi} u_{8}^{3 / 2}(\xi) d \xi$.

$$
u_{9}=\frac{3 \sqrt{3 /\left(8 m^{2}-4\right)}}{k^{2}\left(2 m^{2}-1\right)}[m \operatorname{sn}(\xi ; m) \pm i \operatorname{dn}(\xi ; m)]-\frac{3}{2 k^{2}\left(2 m^{2}-1\right)},
$$

where $\lambda=27 /\left[k^{6}\left(2 m^{2}-1\right)^{3}\right]$, and the relationship between $z$ and $\xi$ is $z=\int^{\xi} u_{9}^{3 / 2}(\xi) d \xi$.

Remark 5. The only solution obtained in [20] is just (3.26). One may obtain other types of solutions of (3.16) using (3.21) and other solutions of (1.2).

\subsection{The generalized Pochhammer-Chree(PC) equations}

$$
u_{t t}-u_{t t x x}-\left(a u+b u^{3}+c u^{5}\right)_{x x}=0,
$$

When $a=1, b=1 / 3, c=0$ or $a=1, b=0, c=1 / 5,(3.31)$ reduces to the well-known PC equation[21]. Some solitary wave solutions of (3.31) were also found[22]. Here we will consider its doubly periodic solutions of (3.31). We seek its more types of travelling wave solution in the form $u(x, t)=u(\xi), \xi=k(x+\lambda t)$. Thus (3.31) becomes

$$
k^{2} \lambda^{2} \frac{d^{2} u}{d \xi^{2}}+\left(a-\lambda^{2}\right) u+b u^{3}+c u^{5}=0,
$$


According to Step 2, we make the transformation $u(\xi)=\phi^{1 / 2}$. Thus (3.32) becomes

$$
k^{2} \lambda^{2}\left(2 \phi \phi^{\prime \prime}-\phi^{\prime 2}\right)+4\left(a-\lambda^{2}\right) \phi+4 b \phi^{3}+4 c \phi^{5}=0 .
$$

which has the solution

$$
\phi(\xi)=A_{0}+\frac{A_{1} \sin w(\xi)}{R+P \sin w(\xi)+Q \cos w(\xi)}+\frac{B_{1} \cos w(\xi)}{R+P \sin w(\xi)+Q \cos w(\xi)},
$$

with $w$ satisfying (1.2).

With the aid of symbolic computation, the substitution of (3.34) with (1.6) into (3.33) can determine these parameters $A_{0}, A_{1}, B_{1}, R, P, Q, k, \lambda$. Therefore we have the double periodic solutions of (3.31):

$$
u_{1}=\left\{ \pm \frac{3 b}{8 c} \operatorname{sn}[k(x+\lambda t) ; m]-\frac{3 b}{8 c}\right\}^{1 / 2}
$$

where

$$
\begin{gathered}
\lambda=\sqrt{1 /\left(64 c m^{2}\right)\left(-15 b^{2} m^{2}+64 a c m^{2}+3 b^{2}\right)}, \\
k=\sqrt{-12 b^{2} /\left(-15 b^{2} m^{2}+64 a c m^{2}+3 b^{2}\right)} . \\
u_{2}=\left\{ \pm \frac{3 b m}{8 c} \operatorname{sn}[k(x+\lambda t) ; m]-\frac{3 b}{8 c}\right\}^{1 / 2},
\end{gathered}
$$

where

$$
\begin{aligned}
\lambda & =\sqrt{1 /(64 c)\left(3 b^{2} m^{2}+64 a c-15 b^{2}\right)}, \\
k & =\sqrt{-12 b^{2} /\left(3 b^{2} m^{2}+64 a c-15 b^{2}\right)}, \\
u_{3} & =\left\{ \pm \frac{3 b}{8 c} \operatorname{cn}[k(x+\lambda t) ; m]-\frac{3 b}{8 c}\right\}^{1 / 2},
\end{aligned}
$$

where

$$
\begin{gathered}
\lambda=\sqrt{1 /\left(64 c m^{2}\right)\left(-12 b^{2} m^{2}+64 a c m^{2}-3 b^{2}\right)}, \\
k=\sqrt{12 b^{2} /\left(-12 b^{2} m^{2}+64 a c m^{2}-3 b^{2}\right)} . \\
u_{4}=\left\{i b c^{-1} Z \operatorname{sn}[k(x+\lambda t) ; m]+b c^{-1} Z \operatorname{cn}[k(x+\lambda t) ; m]\right\}^{1 / 2},
\end{gathered}
$$

where

$$
\begin{aligned}
\lambda & =\sqrt{1 /\left(96 c m^{2}\right)\left(-27 b^{2} m^{2}+96 a c m^{2}+\left(64 b^{2} m^{2}+128 b^{2}\right) Z^{2}\right)}, \\
k & =\sqrt{512 b^{2} Z^{2} /\left(-27 b^{2} m^{2}+96 a c m^{2}+\left(64 b^{2} m^{2}+128 b^{2}\right) Z^{2}\right)} .
\end{aligned}
$$

and $Z$ is the solution of equation

$$
4096 m^{2} Z^{4}+81 m^{2}+\left(-1152 m^{2}+2304\right) Z^{2}=0 .
$$

Moreover we also obtain other types of solutions of (3.31) using (3.34) and other solutions of (1.2). We omit them here.

\section{Conclusions}


In summary, we have presented a new algorithm to seek both solitary wave solutions and doubly periodic solutions of nonlinear wave equations based the sine-Gordon reduction equation (1.2) and the transformation (2.2). We choose the double sine-Gordon equation, the Magma equation and the generalized Pochhammer-Chree(PC) equation to illustrate the method. As a result, many types of new solitary wave solutions, singular solitary wave solutions and doubly periodic solutions are obtained. Moreover when using the method to these special nonlinear equations, some transformations are firstly needed.

It is easy to see that we only use the method to consider travelling wave solutions of nonlinear differential equations. To obtain enlarge the scope of solutions. we may further extend the method to be a generalized form, which may be used to seek non-travelling wave solutions.

Let $\xi \rightarrow \psi(x, t)$, then (1.2) becomes

$$
d w(\psi(x, t)) /(d \psi(x, t))=\mu \sqrt{a+b \sin ^{2} w(\psi(x, t))},
$$

where $\psi(x, t)$ is a smooth function of $x, t$. For different parameters $a, b$ we know that (4.1) has the similar solutions to (1.2). For example, in the case $a=1, b=-m^{2}$, (4.1) becomes the first-order ODE

$$
d w(\psi(x, t)) /(d \psi)= \pm \sqrt{1-m^{2} \sin ^{2} w(\psi(x, t))}
$$

which has the solutions

$$
\sin [w(\psi(x, t))]=\operatorname{sn}(\psi(x, t) ; m), \quad \text { or } \quad \cos [w(\psi(x, t))]=\operatorname{cn}(\psi(x, t) ; m),
$$

and

$$
\sin [w(\psi(x, t))]=\operatorname{ns}(\psi(x, t) ; m) / m, \quad \text { or } \quad \cos [w(\psi(x, t))]=i \operatorname{ds}(\psi(x, t) ; m) / m,
$$

For the given nonlinear partial differential equation (2.1), we do not need to firstly make the travelling wave transformation $u(x, t)=u(\xi), \xi=k(x+\lambda t)$ to reduce (2.1) to an ODE with constant coefficients. We directly assume that (2.1) has the generalized formal solution

$$
u(x, t)=u(w(\psi))=A_{0}(x, t)+\sum_{j=1}^{m} \frac{\cos ^{j-1} w(\psi)\left[A_{j}(x, t) \sin w(\psi)+B_{j}(x, t) \cos w(\psi)\right]}{[R(x, t)+P(x, t) \sin w(\psi)+Q(x, t) \cos w(\psi)]^{j}},
$$

where $w(\psi)$ satisfies $(4.1), A_{j}(x, t)^{\prime} s, B_{j}(x, t)^{\prime} s, \psi(x, t), R(x, t)^{2}+P(x, t)^{2}+Q(x, t)^{2} \not \equiv 0$ are functions to be determined later.

The substitution of (4.5) into (2.1) with (4.1) yields a set of nonlinear differential equations in unknowns. Solving the set of equations may can determine these unknowns. It is obvious to see that solving the set of nonlinear differential equations is difficult. But some special forms of these unknowns may be obtained with the aid of symbolic computation. This will be studied further.

\section{Acknowledgments}

This work is supported by the NNSF of China (No. 10401039) and the NKBRSF of China (2004CB318002). 


\section{References}

[1] M.J. Ablowitz and P.A. Clarkson, Solitons, Nonlinear Evolution Equations and Inverse Scattering (Cambridge: Cambridge University Press, 1991).

[2] D. V. Patrick, Elliptic Function and Elliptic Curves, Cambridge University Press, Cambridge, 1973.

[3] E.T. Whittaker and G.N. Watson, Modern Analysis,Cambridge University Press, Cambridge, 1952.

[4] C. T. Yan, Phys. Lett. A, 224(1996)77.

[5] Z.Y. Yan and H.Q. Zhang, Phys. Lett. A, 252(1999)251.

[6] Z.Y. Yan et al., Acta Phys. Sin., 48(1999) 1.

[7] Z.Fu, et al., Phys. Lett. A, 299(2002)507.

[8] Z.Y. Yan, Chaos, Solitons and Fractals, 16(2003) 291.

[9] Z.Y. Yan, Comput. Phys. Commun., 153(2003) 154.

[10] W.X. Ma, et al., Int. J. Non-linear Mech., 31(1996)329.

[11] E.G.Fan, Phys.Lett. A, 277(2000)212.

[12] Z.Y.Yan and H.Q.Zhang, Phys.Lett.A, 285(2001)355.

[13] Z.Y. Yan, J. Phys. A, 36(2003)1961.

[14] E. G. Fan, Chaos, Solitons and Fractals, 15(2003)567.

[15] R. Conte and M. Musette, J. Phys. A, 25(1992) 5609.

[16] Z.Y.Yan, Chaos, Soliton and Fractals, 16(2003)759.

[17] S.Liu, et al., Phys. Lett. A, 289(2001)69.

[18] E.G.Fan and J. Zhang, Phys. Lett. A, 305(2002)383.

[19] D.R.Scott and D.J. Stevenson, Geophys. Res. Lett., 11(1984) 1161.

[20] D. Takahashi and J. Satsuma, J. Phys. Soc. Jpn., 57(1988)417.

[21] I.L. Bogolubsky, Comput. Phys. Commun., 13(1977)149.

[22] W.G. Zhang and W.X. Ma, Appl. Math. Mech., 20(1999)625. 